\title{
Draft genome of the Brazilian railroad worm Phrixothrix hirtus E.Olivier (Phengodidae:
}

\section{Coleoptera)}

Danilo Trabuco Amaral ${ }^{1,2, \#}$, Yasuo Mitani ${ }^{3}$, Isabel Aparecida Silva Bonatelli ${ }^{4}$, Ricardo Cerri ${ }^{5}$, Yoshihiro Ohmiya $^{6,7}$, Vadim Viviani ${ }^{8,9 *}$

${ }^{1}$ Department of Biology. Center for Human and Biological Sciences. Universidade Federal de São Carlos (UFSCar). Sorocaba, Brazil.

${ }^{2}$ Graduate Program in Comparative Biology. Faculty of Philosophy, Sciences and Languages of Ribeirão Preto. Universidade de São Paulo (USP). Ribeirão Preto, Brazil.

${ }^{3}$ Bioproduction Research Institute, National Institute of Advanced Industrial Science and Technology (AIST), Sapporo, Japan.

4 Department of Ecology and Evolutionary Biology. Universidade Federal de São Paulo (UNIFESP). Diadema, São Paulo, Brazil.

${ }^{5}$ Department of Computational Science, Universidade Federal de São Carlos (UFSCar). São Carlos, Brazil.

${ }^{6}$ Biomedical Research Institute, AIST, Ikeda-Osaka, Japan.

${ }^{7}$ Osaka Institute of Technology, OIT, Osaka, Japan.

${ }^{8}$ Graduate Program of Evolutive Genetics and Molecular Biology, Federal University of São Carlos (UFSCar), São Carlos, Brazil.

${ }^{9}$ Graduate Program of Biotechnology and Environmental Monitoring, Federal University of São Carlos (UFSCar), Sorocaba, Brazil.

*Corresponding author: Rodovia João Leme dos Santos, Km 110, SP264. ZIP 18052-780, Sorocaba, Brazil. Phone: +55-015-3229-5983, Fax: + 55-015-3229-5983. email: viviani@ufscar.br; ${ }^{\#}$ danilo.trabuco@gmail.com

\section{Highlights}

- First draft genome assembly of Phengodidae, the largest one described in Coleoptera;

- Gene family expansions associated with anatomical development and morphogenesis;

- Bioluminescent control and luciferin biosynthesis genes are common within Elateroidea;

- Despite similar bioluminescent system, metabolic routes may have evolved independently; 


\begin{abstract}
The Neotropical region is the richest in bioluminescent Coleoptera species, however, its bioluminescence megadiversity is still underexplored in terms of genomic organization and evolution, mainly within the Phengodidae family. The railroad worm Phrixothrix hirtus is an important biological model and symbolic species due to its bicolor bioluminescence, being the only organism that produces true red light among bioluminescent terrestrial species. Here, we performed the partial genome assembly of $P$. hirtus, combining short and long reads generated with Illumina sequencing, providing an important source of genomic information and a framework for comparative genomic analyses for the evaluation of the bioluminescent system in Elateroidea. The estimated genome size has $\sim 3.4 \mathrm{~Gb}, 32 \%$ of GC content, and $67 \%$ of repetitive elements, being the largest genome described in the Elateroidea superfamily. Several events of gene family expansions associated with anatomical development and morphogenesis, as well as distinct odorant-binding receptors and retrotransposable elements were found in this genome. Similar molecular functions and biological processes are shared with other studied species of Elateriformia. Common genes putatively associated with bioluminescence production and control, including two luciferase genes that displayed 7 exons and 6 introns, and genes that could be involved in luciferin biosynthesis were found, indicating that there are no clear differences about the presence or absence of gene families associated with bioluminescence in Elateroidea. In $P$. hirtus the conversion of $L$ - to $D$ luciferin seems to involve additional steps using a Palmitoyl-CoA thioesterase instead of an Acyl-CoA synthetase, which was found in Lampyridae species.
\end{abstract}

Keywords: Bioluminescence, Elateroidea, Gene families, Genomic DNA, Morphogenesis, Phengodidae. 


\section{Introduction}

The Neotropical region hosts the richest diversity of bioluminescent Coleoptera species in the world (Costa et al., 2010), including species of the three main families of Elateroidea: Elateridae, Lampyridae, and Phengodidae (Viviani, 2002; Rosa, 2010, Oba et al., 2011), and also two species of Staphylinidae (Costa et al., 2986; Rosa, 2010). Despite such richness, the Neotropical species are still poorly studied in terms of taxonomy, ecology, and evolution. Among the three main families, the less studied one is Phengodidae, which has 35 genera and 200 species distributed in three tribes, Pennicilloporini, Phengodini and Mastinocerini, the latter being found predominantly in the Neotropical region (Wittmer, 1976; O’Keefe, 2002; Viviani, 2002). The Phrixothrix hirtus E.Olivier (1909) railroad worm (Fig. 1a) is certainly the most spectacular example of bioluminescence in Phengodidae, with its unique red-light emitting cephalic and post-cephalic lanterns and yellow-green emitting lanterns in the body, having been used as an important biological model for biochemical and molecular studies of luciferases (Viviani et al., 1999a; 2001; 2004; 2013; 2021; Viviani and Ohmiya, 2000; Amaral et al., 2016; Bevilaqua et al., 2019). The bicolor bioluminescence is caused by the presence of luciferase isozymes in the head and lateral body lanterns (Viviani and Bechara, 1995), being an example of paralogy caused by events of gene duplication in Mastinocerini (Arnoldi et al., 2010).

Genome analysis has been used to understand the evolutionary processes that drive the diversification and the evolution of the bioluminescence in Elateroidea (Braham and Wenzel, 2001; Day et al., 2004; Amaral et al., 2019a; 2019b). The genome size estimation in Elateroidea based on flow cytometry was also performed for all bioluminescent families (Hanrahan and Johnston, 2011; Liu et al., 2017; Lower et al., 2017). These studies suggested that the genome sizes among species of this superfamily range from $0.4 \mathrm{~Gb}$ to $2.2 \mathrm{~Gb}$, and identified a positive relationship between the genome size and the amount of repetitive DNA.

Recently, the genome sequence and assembly of Photinus pyralis firefly (Lampyridae) and Ignelater luminosus click-beetle (Elateridae) (Fallon et al., 2017; Fu et al., 2017; Andrews et al., 2020) were conducted and deposited in public genetic database repositories. These genomic analyses showed the divergence of the ancestral luciferase, supporting the independent origins of bioluminescence in Elateridae and Lampyridae. They also showed the existence of two distinct luciferase genes in P. pyralis firefly located in two chromosomes, suggesting events of gene duplication associated with a translocation between the chromosomes, which corroborates the presence of two luciferases isozymes in the lanterns and fat body in distinct life stages of fireflies (Strause and DeLuca, 1981; Viviani et 
al., 2008; Oba et al., 2010; Bessho-Uehara and Oba, 2017; Carvalho et al., 2020). In addition, genomic and transcriptomic analyses of two Palearctic firefly species, Abscondita terminalis and Lamprigera yunnana, indicated putative luciferin biosynthesis pathways in fireflies (Zhang et al., 2020), involving several gene products, which were also described in RNA-Seq analyses in other Elateroidea species (Vongsangnak et al., 2016; Amaral et al., 2017a,b;2018). Such studies brought important contributions and insights about the genome organization within Elateroidea, however, the functional genomic information remains limited to Lampyridae and Elateridae.

However, with the exception of RNA-Seq analysis of the lateral lanterns and fat body of $P$. hirtus which identified several luciferase-like enzymes in these tissues and a luciferase enzyme in photogenic tissues (Amaral et al., 2017a; 2017b; 2019b), no genome sequence analyses was conducted for the Phengodidae family yet. Genome sequence analysis in this family may improve the knowledge about the origin and evolution of the family, as well as the origin and control of bioluminescence.

Here we report the first draft genome assembly of a Phengodidae species, the SouthAmerican Phrixothrix hirtus E.Olivier railroad worm, using both genomic short-read and mate-pair libraries. This species displays the largest genome among Elateriformia species studied so far, as well as the presence of several transposable element families. With this draft genome, we produced a partial genome assembly, which is a novel important source of information for future structural, genetic, evolutionary studies, and for comparative genomic analyses in Coleoptera species evolution of bioluminescence in Elateroidea.

\section{Results and Discussion}

\section{1. de novo Genome Assembly and Annotation}

The genome of $P$. hirtus (Fig. 1a) is the seventh sequenced and available genome within the Elateroidea superfamily and the first one in the Phengodidae family. The phylogenetic analysis showed a strict relationship between Lampyridae and Phengodidae (Fig. 1b). Based on previous findings, Phengodidae diverged from the sister Palearctic family, Rhagophthalmidae, around 73.4 Ma, and from Lampyridae around 97.3 Ma (Amaral et al., 2019). The ultrametric tree performed by the r8s software suggested that the divergence between Phengodidae and Lampyridae occurred even earlier, around $114 \mathrm{Ma}$.

Here, we generated and assembled $\sim 190 \mathrm{~Gb}$ (56.8-fold coverage) from Illumina short reads and $\sim 65 \mathrm{~Gb}$ (20.2-fold coverage) from Illumina mate-pair reads (Table S1 and S2). The assembly length, $\sim 3.40 \square \mathrm{Gb}$, was consistent with the $k$-mer estimate genome size $(\square 3.4 \square \mathrm{Gb}$ 
in Jellyfish2.0 and $\sim 3.35 \mathrm{~Gb}$ in GenomeScope; Fig. S1). So far, this is the largest described genome among Elateroidea species (from $0.42 \mathrm{~Gb}$ to $2.5 \mathrm{~Gb}$ ) and also in Coleoptera (from $0.15 \mathrm{~Gb}$ to $2.7 \mathrm{~Gb}$ ) (Hanrahan and Johnston, 2011). Previously, we showed that the mtDNA genome of $P$. hirtus is also larger than those from other Elateroidea species, with duplication events and a larger control region (Amaral et al., 2016). Several hypotheses were suggested and tested to evaluate the genome size correlation in Coleoptera, including body size (morphological; Palmer and Petitpierre, 1996), chromosome number (Petitpierre et al., 1993), methylation rate (Lechner et al., 2013), and reproductive fitness (Arnqvist et al., 2015). However, the genome size correlation in railroad worms needs to be better explored, including new species and morphological, environmental, and genomic data.

The $P$. hirtus scaffolded genome is detailed in table $\mathrm{S} 2$. The gene prediction using Augustus resulted in 253,925 gene models in the first round of analysis, and 92.234 gene products in the second round of analysis. The predicted proteins were found against the SwissProt database, in which 87,967 genes were assigned to the putative function (Fig. S2). These observed GO terms are common in most arthropods, being responsible for basic physiologic processes and metabolic activities that are essential in Insecta. We did not identify any specialized function occurring only in $P$. hirtus. The genome contains approximately $70 \%$ complete single-copy orthologs and multi-copy orthologs, which indicate that most parts of genes were recovered. The percentage of observed GC content was $\sim 32 \%$ and the estimated heterozygosity was about $0.25 \%$ (Fig. S1). We obtained a total of $\sim 12$ million elements of repetitive DNA $(2,326,217,477 \mathrm{bp}$, or $2.4 \mathrm{~Gb})$, representing approximately $67 \%$ of the total length of the assembled scaffolds (Table S3).

We also completely assembled the circular mitogenome of $P$. hirtus, which displayed 20,303 bp, similarly to the previously studied in this species (Amaral et al., 2016), again being the largest Elateroidea mtDNA described. The mtDNA presented 2 ribosomal RNAs (rnaS and rnaL), 13 protein coding-genes (PCG), and 21 tRNAs (absence of tRNA-A; Table S4). A larger $A+T$ rich-region (5,661 bp), which includes four partial copies of the ND2 gene and the tRNA-Q was observed. Similar to the nuclear genome, these results also support the evidence of high dynamism of the $P$. hirtus genomes.

\subsection{Repetitive element DNA content}

The inter-species genome size variation could be the result of several gene/genome duplication and/or deletion events (Blommaert, 2020), however, the rearrangements and duplication of repetitive DNA sequences may also imply large genomes sizes (Talla et al., 
2017). Studies are showing the role of these elements as potential substrates for new genes and their association with gene expression (e.g. epigenetic regulation), as well as a stress response (regulatory sequence) (Rech et al., 2019; Choi and Lee, 2020; Fedoroff, 2020). Thus, these elements could be major drivers of genome evolution in Eukaryotes (Quesneville, 2020).

According to BUSCO, there was no excess of duplicated genes (7.42\% of duplicates). Nevertheless, the analysis of the landscape of repetitive and transposable elements (TE; Table S3) of $P$. hirtus showed that its genome has a great part of base-pair length composed by repetitive elements $(\sim 67 \% ; 2.4 \mathrm{~Gb})$. The percentages of TE content in Elateroidea vary from $19.8 \%(\sim 190 \mathrm{Mb})$ in A. lateralis to $42.6 \%(\sim 180 \mathrm{Mb})$ in $P$. pyralis (Fallon et al., 2018). In $P$. hirtus, the majority of these TE in P. hirtus were unclassified (46.83\%) followed by the DNA transposons (12.3\%). Recent studies have also discussed the variation of TE contents among Coleoptera, Diptera (6\% in the Belgica antarctica to $58 \%$ in Anopheles gambiae), Hymenoptera (less than 6\% in Apis mellifera and Athalia rosae), and Orthoptera (58\% in Locusta migratoria), which suggested that the repetitive elements are highly dynamic in Insecta (Hazzouri et al., 2020).

\subsection{Orthologous Analysis and Evolution of gene families in Elateriformia}

The comparative genomic analysis evaluates the ortholog gene families represented in the genome to improve the understanding of the evolution within this superfamily. Here, the orthologous analysis using the amino acid sequences of Elateriformia species (Abscondita terminalis, Lamprigera yunnana, Photinus pyralis (Lampyridae), Ignelater luminosus, Limonius californicus (Elateridae), and Agrilus planipennis (Buprestidae)) against P. hirtus showed 17,862 ortholog gene families. Among them, 2,001 orthogroups (11.2\% of the total) were commonly shared among all species (Fig. 2), with only 359 orthogroups displaying a single gene copy, which were applied to phylogenetic reconstruction (Fig. 1b); a total of 523 orthogroups in P. hirtus did not share direct orthology with the other six protein species. The bioluminescent species used in this study shared 2,477 orthogroups, however, only 440 displayed single-copy genes.

The molecular function ontology of the predicted gene products shared among all Elateriformia species shows enrichment of catalytic activity and binding enzymes (Fig. S3A and S3B). Among catalytic activity, the hydrolase and transferase were highlighted, while among binding functions, ion-binding, heterocyclic binding, organic cyclic compound binding, and protein binding were highlighted (Table S5). Biological processes of the shared 
GO were mostly enriched for cellular processes (regulation of cellular process and cellular metabolic process), biological regulation (regulation of biological quality), regulation of biological process (regulation of metabolic process), and metabolic process (nitrogen compound metabolic process, organic substance metabolic process, and primary metabolic process) (Fig. S3A and S3C).

We separately evaluated the orthogroups associated only with the bioluminescent species of the Elateroidea (Table S6). Considering these five species, we obtained 2,477 orthogroups, whereas 476 were exclusively for bioluminescent individuals. We also annotated their gene ontology (Fig. S4). Here, we identified several families of general odorant-binding proteins (OBP), responsible for recognizing and transporting hydrophobic odorants to the antennal sensilla and activating the olfactory signal transduction pathway ( $\mathrm{Li}$ et al., 2016). For many flashing firefly species such as Photinus pyralis, it is expected and suggested that the luminescence emission pattern (flash, continuum/glow, etc.) is the main factor responsible for intraspecific communication. However, in Phrixothrix railroad worms, as well as other Phengodidae, it is known that pheromone detection plays a major role in sexual attraction (Jacobson, 2012). The presence of these several OBP gene families supports that both the pheromones and bioluminescence are involved in the communication between Elateroidea species.

The comparison analysis among Elateriformia and Elateroidea did not show clear differences in the presence or absence of gene families among bioluminescent species and their closely related non-bioluminescent species, with the exception of OBP gene families in Phengodidae. These results suggest that both bioluminescent and non-bioluminescent species display similar groups of gene families, including luciferase and/or luciferase-like enzymes, genes associated with luciferin biosynthetic pathway (Niwa et al., 2006; Oba et al., 2013; Amaral et al., 2017a; 2017b; 2019b; Zhang et al, 2020), sulfotransferase (Fallon et al., 2016), etc. However, the transcription control and expression level of these genes could be the main determinants of the spatial and temporal control of the bioluminescence in Elateroidea, rather than a genomic feature.

\subsection{Analysis of unique $P$. hirtus orthogroups}

We observed 523 orthogroups in P. hirtus that did not share direct orthology with the other studied species. In the annotation of these gene families, however, only a small part of the orthogroups was assigned ( 12\%) (Table S7). From them, several retrotransposable elements, such as LINE, MOS1, ATP Translocases, and PiggyBac, were present among these 
orthogroups. The high amount of these elements exclusively in the P. hirtus genome may explain the largest genome. The eukaryotic genome is highly dynamic, including events of gene duplication or even whole-genome duplication (Ting et al., 2004; Van de Peer et al., 2009; Mendivil and Ferrier, 2012). In arthropods, these mechanisms may have important evolutionary significance when associated with adaptation to environmental changes and the processes of biological and cellular regulation and simultaneous response to external stimulus (Kidwell, 2002; Chénais et al., 2012).

We also observed in P. hirtus, the gene families of Craniofacial development protein and Heat Shock, which seem to work in consonance to the development of wing dimorphism in arthropods (Carrol, 1995; Baral et al., 2019; Chen et al., 2019). In Phengodidae, including $P$. hirtus railroad worms, the sexual dimorphism is accentuated, with a clear distinction between male and female body shape and bioluminescent organs (Wing et al., 1984). The females are neotenic and paedomorphic (retention of larval traits), while the males undergo metamorphosis and develop wings in the adult stage. Furthermore, the males are much smaller than the females, besides the presence of wings. However, more molecular biochemical studies are necessary to understand the genes involved in metamorphosis and sexual differentiation in the family Phengodidae.

\subsection{Expansion of ortholog gene families}

In the past few years, comparative genomic studies showed the dynamic aspect of genome size and gene families in rapidly evolving groups of species, such as plants and insects (Zhang et al., 2018; Freitas and Neri, 2020; Hazzouri et al, 2020; Jiao, et al., 2020; Wang et al., 2020). The expansion and contraction of gene families seem to be pervasive and provide evidence that the copy number changes are associated with the natural selection acting under the particular adaptation of the species, such as changes in protein-coding and regulatory regions (Hahn et al., 2009). These gene families with complex gene duplication histories in lineages deserve great attention. Thus, we estimated the expansion and contraction of gene families among Elateriformia species.

The protein orthogroups obtained here were managed to identify signatures of expansion in gene families among Elateriformia. The number of rapidly evolving, expansions, and contractions gene families was displayed in Fig. 3. There are a total of 2,134 expanded and 7,581 contracted gene families among Elateroidea families internal branches. The largest number of contraction gene families was found in P. hirtus $(9,783)$, while the largest number of expansion was found in Limonius (4,268). The branches with the largest 
numbers of rapidly evolving gene families are both leading to Elateridae and Lampyridae families (194 and 173, respectively). The ancestor branch of Elateroidea displayed 1,514 and 547 gene families with contractions and expansions, respectively. Details about the molecular function and biological processes of the gene families that displayed expansion within the Elateroidea superfamily are shown below.

Elateroidea. In the main branch of Elateroidea, we observed 547 expanded gene families. From them, a total of 465 gene families were annotated and classified concerning their molecular function (Table S8). The gene families are involved with catalytic activity (hydrolase and oxidoreductase activity) and binding (ion, organic cyclic compound, and heterocyclic compound binding), as well as metabolic processes (organic substance, nitrogen, and primary metabolic process), regulatory processes, and response to stimuli (Fig S5A). We found a high amount of hydrolases (several lipase members, serine hydrolase, glutathione hydrolase, Acyl-CoA thioesterase), transferases (glutathione S-transferase, UDPglucosyltransferase, Acyl-coenzyme A:cholesterol acyltransferase 2, Sulfotransferase, odorant binding), ligase (E3 ubiquitin ligase), and oxidoreductases (several Cytochrome P450 members, Fatty acyl-CoA reductase, luciferase, phenoloxidase). Several of these gene families in the expansion are associated with detoxification and metabolism of xenobiotics (cytochrome P450s, glutathione S-transferases, UDP-glucuronosyltransferases, etc.) (Ahn et al., 2012; Zhu et al., 2016; Rane et al; 2019), and were widely identified among the transcriptome dataset of Elateroidea (Amaral et al., 2017a; 2017b; 2019b). These genes play fundamental roles in xenobiotic detoxification and degradation of distinct molecules related to an insect diet, detoxification of metabolic compounds, resistance to pesticides, degradation of hormones (Xue et al., 2020), and the degradation of the lucibufagins (defensive steroids), present in some fireflies species and used for defensive purposes (McKinley and Lower, 2020).

Elateridae. In the Elateridae branch, we identified and annotated 819 gene families (from 1,170; Table S9), which recovered quite similar patterns observed to the Elateroidea branch, with the high number of gene families related to the catalytic activity (hydrolase activity) and biding (ion, organic cyclic compound, and heterocyclic compound binding) and metabolic processes (organic substance, nitrogen, and primary metabolic process). We also observed a high amount of cellular processes (regulation and metabolic process; Fig S5B). Gene families such as lipase members, serine hydrolase, glutathione S-transferase, UDPglucosyltransferase, Sulfotransferase, luciferase, E3 ubiquitin ligase, several Cytochrome P450 members, and Fatty acyl-CoA reductase were the most common gene identified. 
However, we also observed the expansion of phenoloxidase 1 gene family, succinate CoAligase (ADP-formin superfamily), and craniofacial development protein 2 (relevant to wingshed occurrence; Chen et al., 2019).

Agrypininae/Pyrophorini (bioluminescent click beetles Ignelater luminosus). We evaluated the gene families' expansion within the bioluminescent species Ignelater luminosus of Elateridae and obtained 1,856 gene families. A total of 1,366 were annotated (Table S10). The GO molecular function and biological processes were quite similar to the Elateridae branch (Fig S5C). Several ubiquitin protein ligases, sulfotransferase (five distinct gene families), Cytochrome P450, UDP-glucosyltransferase, and glutathione S-transferase were identified. Evaluating all other branches, the expansion of the luciferase gene family is present only in Elateridae, with the presence of several luciferases and luciferase-like enzymes (Fallon et al., 2018). The high amount of replication and transcription factors, perithophin (insect pathogenic protection), and keratin-associated protein (also observed in Lampyridae) were singular to the I. luminosus.

Denticollinae (Non-luminescent Elateridae L. californicus). The non-luminescent click beetle Limonius displayed the highest number of expanded gene families among the studied species (4,268 with 2,146 annotated; Table S11). These gene families are associated with the binding molecular function (mainly ion binding) and regulation, metabolic, and stimulus of cellular processes (Fig S5D). Several families displayed more than 2 or 3 distinct gene families. The interesting finding is the expansion of two distinct families of luciferase, however, we did not identify the expansion of luciferase-like gene family, such as 4coumarate-CoA ligase and succinate CoA-ligase (ADP-formin superfamily), which were present in other genomes and also observed in recent transcriptome analysis luminescent and non-luminescent Elateridae species (Amaral et al., 2019b).

Lampyridae. A total of 256 (from 416) gene families were annotated (Table S12). Major molecular function and biological processes of gene families were catalytic activity, binding, and cellular processes (Fig S5E). We observed the expansion of glutathione Stransferase, sulfotransferase (three distinct gene families), acyl-CoA thioesterase, and Cytochrome P450, which are highlighted in other Elateroidea species. Here, we did not identify the expansion of the luciferase gene family, however, we observed the expansion of the 4-coumarate-CoA ligase, which also belongs to the adenylate-forming family. This group of gene families was also observed in the transcriptome analysis of the three bioluminescent species of Elateroidea, Elateridae, Lampyridae, and Phengodidae (Amaral et al., 2017a, 2017b, 2019b). We identified the expansions of the superoxide dismutase (SOD), 
cystathionine-B-synthase, and cysteine-rich protein 2-binding. The SOD enzyme seems to be associated with the protection of lanterns during hyperoxia and oxidative stress and is recovered in high abundance in lanterns of firefly species (Barros and Bechara, 1998; Amaral et al., 2017b). The other two gene families are associated with cysteine availability, which is a precursor of luciferin biosynthesis in Elateroidea (Viviani et al., 2013; Kanie et al., 2016; Amaral et al., 2017a, 2017b, 2019b; Zhang et al., 2020).

Phengodidae (Phrixothrix hirtus). From the 1,491 gene families expanded within $P$. hirtus, a total of 1,346 were annotated (Table S13). We observed the presence of several transferases, hydrolases, and ligases gene families, which were also identified in the other Elateroidea species. However, we recovered distinct unique or overrepresented molecular functions and biological processes in P.hirtus (Fig. S5F), such as multicellular organismal development, anatomical structure development and morphogenesis, reproduction, and multiorganism process (negative regulation of metabolic process). These terms are associated with the ontogenetic evolution of the organism, orchestrating the complex processes associated with embryogenic and growth development (Hill et al., 2010). The neoteny of the Phengodidae females and the winged male metamorphosis could be related to those gene families' expansion associated with organismal ontogeny. The superoxide dismutase gene family was also expanded in Phengodidae, as well as hypoxia-inducible factor 1-alpha, which plays a role in the cellular response to $\mathrm{O}_{2}$ deprivation (Harrison et al., 2018). Several gene families displayed distinct orthologs in P. hirtus, such as axin-1 like (anterior development in Coleoptera; Fu et al., 2012), chromobox protein homolog (crucial in the establishment and maintenance of heterochromatin in larvae; Shoji et al., 2013), doublesex- and mab-3-related transcription factor (Sex determination mechanisms and sex differentiation; Rather and Dhandare., 2019), forkhead box (insulin signaling pathway and regulate physiological processes and juvenile hormone degradation; Zeng et al., 2017). Some other physiological mechanisms displayed overrepresented genes and we briefly discussed them below.

Extracorporeal digestion. The extracorporeal digestion is observed among distinct Insect families larvae, including the superfamily Elateroidea, in which this process was described in larvae of all bioluminescent species (Colepicolo-Neto et al. 1986; Bechara, 1988; Cohen et al., 1995). In the field, the Phengodidae larvae prey on millipede species (with equal or bigger corporeal size) or termites, introducing the digestive cocktail directly to the organism and, posteriorly, ingesting the partially digested fluid (Viviani and Bechara 1997). Colepicolo-Neto et al. (1986) showed a similar process in P. termitilluminans larva (Elateridae), identifying the presence of distinct proteases and carbohydrate hydrolases in the 
regurgitated fluid. Here, we identified the expansion of gene families associated with protease and carbohydrate hydrolases and extra-oral digestion in Arthropods, such as chymotrypsin-1, chitinase, cathepsin Z, aminopeptidase $N$, and hyaluronidase. These enzymes are also observed in other organisms, such as spiders (Fuzita et al., 2016; Walter et al., 2017). Some of these gene products are also present in the venom component of Heteropteran species and others (Fry et al., 2009; Walker et al., 2016). In particular, hyaluronidase seems to have an important role to increase the permeability of the tissue to the other toxins and digestive proteins (Walker et al., 2016).

Chemoreception. We identified olfactory, gustatory, odorant, and ionotropic receptors in expansion within the $P$. hirtus genome, suggesting the important role of intraspecific chemical communication in the Phengodidae. The chemosensory gene families expanded in the P. hirtus genome are associated with gustatory and odorant receptor 22, olfactory receptor 2AG1, Glutamate receptor subunit 1, and Glutamate receptor ionotropic, kainate 2. The chemosensory genes, in insects, are involved with mating, feeding, coordinating actions (e.g. attack, defense, escape), among others (Yuvaraj et al., 2018; Blomquist and Ginzel, 2021), being important physiological and ecological processes during the speciation process (mate isolation from its closely related species; Wu et al., 2019). The odorant and gustatory receptors are able to detect volatile chemicals, such as pheromones, which could be responsible for intraspecific communication, including mate. In Phengodidae, where the female is neotenic and the winged male displays a well-developed antenna (Costa et al., 1999), chemosensory communication is critical for sexual attraction. However, we did not observe the expansion of the protein binding gene families, the pheromones and odorant carriers, which indicates distinct evolutionary steps among the receptor and binding mechanisms in Phengodidae.

\subsection{Gene families involved in $\boldsymbol{P}$. hirtus bioluminescence}

Based on previous molecular analysis, involving genomic and transcriptomic data, and biochemical studies (Niwa et al., 2006; Viviani et al., 2013; Oba et al., 2013; Kanie et al., 2016; Vongsangnak et al., 2016; Amaral et al., 2017a; 2017b; 2019b; Fallon et al., 2018; Zhang et al., 2020), we looked for specific described gene products that could be involved in the bioluminescence process in Elateroidea, mainly in P. hirtus. Here, we focused on luciferase evolution, bioluminescence emission control, antioxidant enzymes, and the luciferin biosynthesis pathway. 
Luciferase evolution in Phengodidae. The number of AMP-forming enzymes observed in the Elateroidea species is abundant (ca. 15), including bioluminescent and nonbioluminescent species. In the last few years, transcriptomic and genomic data were able to recover the distinct isoforms of luciferase and luciferase-like enzymes and the comparison between their primary amino-acids sequence demonstrated the relationship among these isoforms and their evolution in Elateroidea. The presence of luciferase isozymes in the cephalic and lateral lanterns of Mastinocerini larvae was first shown by Viviani and Bechara (1993). Arnoldi et al. (2010), evaluating the relationship between the luciferase isoforms of Mastinocerini tribe species (Phengodidae), showed the presence of two luciferases, one in the cephalic lantern and one in the lateral body lanterns. These enzymes seem to be more closely related to the same lantern of different species than to the distinct lanterns of the same individual, suggesting an event of gene duplication and paralogy. Here, we identified 17 genes with similarities to luciferase and luciferase-like enzymes.

We were not able to completely assemble the genomic regions that contain the luciferase gene (only partial fragments). Thus, the raw reads were mapped against the Phengodidae luciferase and concatenated them. Using this strategy, we identified two genes that displayed $90 \%$ of similarity to Phengodidae luciferases, which were named PhLuc1 and PhLuc2. The similarity to the luciferase isoform described by Amaral et al. (2017) was $\sim 80 \%$, which may suggest a non-specific gene assembly (possible chimera). The gene lengths for PhLuc1 is 2,019 bp (more similar to the lateral lanterns luciferase), while for PhLuc2 is 2,220 bp (more similar to cephalic lanterns luciferase), both genes comprising seven exons and six introns, which is a similar number of intergenic components observed for the luciferase of Photinus pyralis firefly (Fallon et al., 2018) and Pyrophorus plagiophthalamus click beetle (Elateridae; Feder and Vélez, 2009). The possible duplication event that originated both putative luciferases was followed by a dynamic structural genomic change, which altered the intron size, mainly the introns 1,3 , and 6 , however, we did not observe evidence of intergenic combination. The average size of exons and introns were $\sim 230 \mathrm{pb}$ and $\sim 100 \mathrm{pb}$, respectively.

Bioluminescence emission control and antioxidant enzymes. Recent studies using transcriptome and genomic data described possible gene products associated with the bioluminescent control in Elateridae, Lampyridae, and Phengodidae (Amaral et al., 2017a; 2017b; 2019b; Zhang et al., 2020). Trimmer et al. (2001) and Amaral et al. (2017b) suggested the importance of nitric oxide synthase and octopamine/dopamine receptors to firefly flash control, mainly in the adult stage, in which the flash pattern and duration are fundamental for 
intraspecific communication. However, these gene products were less abundant in Elateridae and Phengodidae, which is consistent with the lower degree of neural control and more continuous glow pattern in these species (Amaral et al., 2017a; 2019b). Here, only a gene of nitric oxide synthase and dopamine/octopamine receptor were found. In Lampyridae genomes, only a copy of the nitric oxide synthase gene was also identified, suggesting a unique gene associated with the control of available oxygen concentration inside the photocytes (Trimmer et al., 2001). The number of dopamine/octopamine receptors was between 8 to 10 copies, much higher than observed in phengodids, consistently with the need for flash control in the adult stage of fireflies.

The presence of catalase and superoxide dismutase in photophores of Elateroidea was previously described by Barros and Bechara (1998) and was associated with detoxification reactive oxygen species (ROS) inside the cells. We already described the abundance of these gene products in the Elateroidea lanterns using a transcriptome dataset and, in this study, we identified two genes of catalase and three of superoxide dismutase Cu-Zn (SOD). The SOD enzyme catalyzes the dismutation of the superoxide radicals to molecular oxygen and $\mathrm{H}_{2} \mathrm{O}_{2}$, while the catalase dismutates the $\mathrm{H}_{2} \mathrm{O}_{2}$ into $\mathrm{O}_{2}$, avoiding harmful damage to the photocytes (Tainer et al., 1983; Cox et al., 2011).

Luciferin biosynthesis. In $P$. hirtus genome, several gene products that were previously potentially associated with luciferin biosynthesis in luminescent Elateroidea were found (Niwa et al., 2006; Viviani et al., 2013; Vongsangnak et al., 2016; Amaral et al., 2017a, 2017b, 2019b; Zhang et al., 2020). Among them, the adenosylhomocysteinase and cysteine sulfinic acid decarboxylase are associated with the conversion of homocysteine to cysteine, which may spontaneously react with $p$-benzoquinone to generate luciferin (Kanie et al., 2016). The gene products involved with tyrosine metabolism (tyrosine aminotransferase, tyrosine hydroxylase, 4-hydroxyphenylpiruvate dioxygenase, and homogentisate 1,2dioxygenase) and the cascade reaction of the L-DOPA pathway (dopamine/octopamine receptor, dopamine $\mathrm{N}$-acetyltransferase, sodium-dependent dopamine transporter, and phenoloxidase and phenoloxidase activating factor) were also observed, as well as a luciferin-regenerating enzyme gene (LRE), which was suggested to recycle the oxyluciferin to an intermediary compound of L-Luciferin (2-cyano-6-hydroxybenzothiazole), and a gene of luciferin sulfotransferase, which converts luciferin to a stable storage compound, sulfoluciferin (Fallon et al., 2016). However, we did not recover any Acyl-CoA thioesterase (ACT) gene which could be involved in the conversion of L-luciferin to D-luciferin (Niwa et al., 2006), whereas we observed the expansion of this gene family among Lampyridae. 
Rather, we identified two genes of palmitoyl-protein thioesterase (a specific group withn ACT), which is a lysosomal enzyme that removes fatty acyl groups from cysteine residues (Glaser et al., 2003), similar to the function of the ACT genes in peroxisomes (Lousa et al., 2013).

These results suggest a quite similar luciferin biosynthetic pathway within Elateroidea bioluminescent species, however, some steps or genes involved in the cascade reactions may have evolved differently during the diversification of the Elateroidea families. Thus, the cellular and metabolic processes may have favored one enzyme over another, such as the palmitoyl-protein thioesterase instead of the Acyl-CoA thioesterase.

\section{Concluding remarks}

We combined short and long reads generated with Illumina sequencing to generate the first draft genome of the South-American Phrixothrix hirtus railroad worm, the first one of the Phengodidae family. This is the largest genome observed in the Elateroidea superfamily, with more than $60 \%$ of its size populated by TE, including the presence of several retrotransposable elements, such as LINE, MOS1, and PiggyBac. The P. hirtus genome displays unique gene families related to anatomical development and morphogenesis, consistently with the neoteny and strong sexual dimorphic development in this species.

The comparison of gene families orthogroups among Elateriformia showed quite similar molecular functions and biological processes shared among all species, which are enriched by catalytic activity and binding enzymes, but did not show clear differences in the presence or absence of gene families associated with the bioluminescence among species/families. A very similar pattern of genes putatively involved in luciferin biosynthetic pathway within Elateroidea bioluminescent species was observed, with the exception of the esterase involved in the conversion of $L$ - to D-luciferin, which is a palmitoyl-thioesterase in $P$. hirtus instead of an acyl-esterase as found in Lampyridae. These results suggest that genes involved in bioluminescence production may have evolved before the divergence within the Elateriformia, whereas the spatial and temporal transcriptional and expression control levels may have evolved later determining the bioluminescence anatomical pattern distribution and control rather than the genomic features.

\section{Material and methods}

\subsection{Sampling, DNA extraction, and libraries construction}


One larval individual of $P$. hirtus was manually collected at "Fazenda Santana" (Souza, Campinas/SP, Brazil) and identified by Prof. Dr. Vadim Viviani. The sample was stored at $-80^{\circ} \mathrm{C}$ until the DNA extraction. The genomic DNA was extracted from whole larvae using DNeasy blood \& tissue kit (Qiagen, USA), according to the instructions of the manufacturer. The DNA quantity and quality were measured using NanoVue (GE HealthCare) and Qubit 3.0 fluorometer (ThermoFisher, USA), and the integrity was checked in agarose gel. Two genomic DNA libraries were prepared: i. using the TruSeq DNA PCRfree library prep kit with fragments of $150 \mathrm{bp}$ and ii.) Nextera Mate Pair library prep with fragments of 2,000 bp (Illumina, USA). The short-read paired-end libraries were sequenced in two independent lanes and the mate-pair library in one lane using the Illumina HiSeq4000 platform (Illumina, USA). The library's construction and sequencing were performed by Hokkaido System Science Co. (Sapporo, Hokkaido, Japan). The raw read data and final genome assembly of $P$. hirtus are deposited and available at the BioProject PRJNA741915.

\subsection{Pre-processing data, de novo genome assembly, and annotation}

The reads obtained by the DNA-Seq were checked by FastQC v0.11.6 software (http://www.bioinformatics.babraham.ac.uk/projects/fastqc/), and adaptors and low-quality reads (Phred Q $\leq 30$ ) were removed using FASTX-TOOLKIT v0.0.14 (Pearson et al., 1997) for the paired-end library and NxTrim (O'Connell et al., 2015) for the mate-pair library. We used DeconSeq v.0.4.3 (Schmieder and Edwards, 2011) software and the RefSeq database (bacteria and viruses) (accessed in April 2018) to remove any microbial contamination within the raw data sequencing. After these filtering processes, we proceed to downstream genomic assembly.

We estimated the best k-mer length for the $P$. hirtus genome assembly using KmerGenie 1.705 (Chikhi and Medvedev, 2014), which was k=81 (data not shown). The genome coverage, size heterozygosity were estimated with estimate_genome_size.pl script (available at https://github.com/josephryan/estimate_genome_size.pl), Jellyfish2 v.2.2.3 (Marcais and Kingsford, 2011) (parameters: count -t $8-C-m 21$--min-quality=20 --qualitystart=33), and GenomeScope2 (Vurture et al., 2017; available at http://qb.cshl.edu/genomescope/genomescope2.0/). We used the gatb-minia-pipeline (available at https://github.com/GATB/gatb-minia-pipeline) for the de novo assembly using 
default settings and fixing k-mer size in 81 . The scaffolded statistics were evaluated using QUAST 5.0.0 (Gurevich et al., 2013). For the mitochondrial genome assembly, we used GetOrganelles software (Jin et al., 2020), with the default setting, and MITOS Web Server (Bernt et al, 2013) for the annotation.

The gene prediction was performed using two-round training in MAKER software with the default settings, combined with transcriptome-based nucleotide and amino acid sequences generated by Amaral et al. (2017a) to improve the prediction. Repetitive DNA elements were identified and annotated using RepeatModeler v.1.0.8 (see http://www.repeatmasker.org/RepeatModeler), RepeatMasker $\quad$ v.4.0.9 (see http://www.repeatmasker.org/). The annotation of the coding regions was conducted using the tool BLASTX against the Swissprot database (retrieved on 05/2020). The GO terms were plotted and visualized using WEGO2.0 (Ye et al., 2018). The completeness of the genes was estimated using BUSCO v.3.0.2 software (Simão et al., 2015).

\subsection{Orthologous protein clustering, gene family evolution, and phylogenetic analysis}

Available genomic data from seven Elateriformia species (details in Table S14) were utilized to perform comparative genomic analyses. The orthogroups identification and the expanded and contracted gene families were identified with OrthoFinder v.2.0.9 (Emms and Kelly, 2015) and CAFÉ v.4.2.1, using birth and death rate (Han et al., 2013), respectively. The ultrametric tree used to determine expansion and contraction family size was performed using r8s v.1.81 software (Sanderson, 2003). The phylogenetic relationship topology was estimated using 359 conserved single-copy orthologs in IQtree2 (Minh et al., 2020) in 1,000 ultrafast bootstraps.

\subsection{Luciferase genes identification}

To identify the putative luciferase gene length, the raw reads were mapped against the luciferase of Phengodidae species available at public databases, using the bowtie2 v.2.4.3 tool (Langmead and Salzberg, 2012). We processed the reads using Samtools v.1.9 (Li et al., 2009) and concatenated them using CAP3 v.10.2011 (Huang and Madan, 1999) software.

\section{Acknowledgments}


We thank the Fundação de Amparo à Pesquisa do Estado de São Paulo (FAPESP grant 2010/05426-8 to VV; FAPESP n. 2017/207340 and 2014/20176-9 to DTA) for Financial Support.

\section{Author contributions}

VV, YO, YM, and DTA conceived the idea; DTA and IASB led both the analyses and manuscript writing; VV, YO, YM, and CR collaborated in manuscript writing. All authors contributed to the intellectual development of the paper, made multiple revisions, and approved the final draft.

\section{Competing interest}

The authors declare no competing interest.

\section{Reference}

Ahn, S. J., Vogel, H., \& Heckel, D. G. (2012). Comparative analysis of the UDPglycosyltransferase multigene family in insects. Insect biochemistry and molecular biology, 42(2), 133-147.

Amaral, D. T., Bonatelli, I. A., Cerri, R., \& Viviani, V. R. (2019a). Phylogenomic analyses and divergence time estimation of Elateroidea (Coleoptera) based on RNA-Seq data. Comparative Biochemistry and Physiology Part D: Genomics and Proteomics, 30, 283289.

Amaral, D. T., Mitani, Y., Ohmiya, Y., \& Viviani, V. R. (2016). Organization and comparative analysis of the mitochondrial genomes of bioluminescent Elateroidea (Coleoptera: Polyphaga). Gene, 586(2), 254-262.

Amaral, D. T., Silva, J. R., \& Viviani, V. R. (2017a). Transcriptional comparison of the photogenic and non-photogenic tissues of Phrixothrix hirtus (Coleoptera: Phengodidae) and non-luminescent Chauliognathus flavipes (Coleoptera: Cantharidae) give insights on the origin of lanterns in railroad worms. Gene Reports, 7, 78-86.

Amaral, D. T., Silva, J. R., \& Viviani, V. R. (2017b). Transcriptomes from the photogenic and non-photogenetic tissues and life stages of the Aspisoma lineatum firefly (Coleoptera: Lampyridae): Implications for the evolutionary origins of bioluminescence and its associated light organs. Gene Reports, 8, 150-159. 
Amaral, D. T., Silva, J. R., \& Viviani, V. R. (2019b). RNA-Seq analysis of the bioluminescent and non-bioluminescent species of Elateridae (Coleoptera): Comparison to others photogenic and non-photogenic tissues of Elateroidea species. Comparative Biochemistry and Physiology Part D: Genomics and Proteomics, 29, 154-165.

Andrews, K. R., Gerritsen, A., Rashed, A., Crowder, D. W., Rondon, S. I., van Herk, W. G., ... \& Hunter, S. S. (2020). Wireworm (Coleoptera: Elateridae) genomic analysis reveals putative cryptic species, population structure, and adaptation to pest control. Communications biology, 3(1), 1-13.

Andrews, S. (2010). FastQC: a quality control tool for high throughput sequence data.

Arnoldi, F. G., da Silva Neto, A. J., \& Viviani, V. R. (2010). Molecular insights on the evolution of the lateral and head lantern luciferases and bioluminescence colors in Mastinocerini railroad-worms (Coleoptera: Phengodidae). Photochemical \& Photobiological Sciences, 9(1), 87-92.

Arnqvist, G., Sayadi, A., Immonen, E., Hotzy, C., Rankin, D., Tuda, M., ... \& Johnston, J. S. (2015). Genome size correlates with reproductive fitness in seed beetles. Proceedings of the Royal Society B: Biological Sciences, 282(1815), 20151421.

Baral, S., Arumugam, G., Deshmukh, R., \& Kunte, K. (2019). Genetic architecture and sexspecific selection govern modular, male-biased evolution of doublesex. Science advances, 5(5), eaau3753.

Barros, M. P., \& Bechara, E. J. (1998). Bioluminescence as a possible auxiliary oxygen detoxifying mechanism in elaterid larvae. Free Radical Biology and Medicine, 24(5), 767-777.

Bechara, E. J. H. (1988). Luminescent Elaterid Beetles. In: Advances in Oxygenated Processes, Vol 1. Greenwich: JAI Press Inc., p. 123-178.

Bernt, M., Donath, A., Jühling, F., Externbrink, F., Florentz, C., Fritzsch, G., ... \& Stadler, P. F. (2013). MITOS: improved de novo metazoan mitochondrial genome annotation. Molecular phylogenetics and evolution, 69(2), 313-319.

Bessho $\square$ Uehara, M., \& Oba, Y. (2017). Identification and characterization of the Luc2 $\square$ type luciferase in the Japanese firefly, Luciola parvula, involved in a dim luminescence in immobile stages. Luminescence, 32(6), 924-931. 
Bevilaqua, V. R., Matsuhashi, T., Oliveira, G., Oliveira, P. S. L., Hirano, T., \& Viviani, V. R. (2019). Phrixotrix luciferase and 6'-aminoluciferins reveal a larger luciferin phenolate binding site and provide novel far-red combinations for bioimaging purposes. Scientific reports, $9(1), 1-17$.

Blommaert, J. (2020). Genome size evolution: towards new model systems for old questions. Proceedings of the Royal Society B, 287(1933), 20201441.

Blomquist, G. J., \& Ginzel, M. D. (2021). Chemical Ecology, Biochemistry, and Molecular Biology of Insect Hydrocarbons. Annual Review of Entomology, 66, 45-60.

Branham, M. A., \& Wenzel, J. W. (2001). The evolution of bioluminescence in cantharoids (Coleoptera: Elateroidea). Florida Entomologist, 565-586.

Carroll, S. B. (1995). Homeotic genes and the evolution of arthropods and chordates. Nature, $376(6540), 479-485$.

Carvalho, M. C., Tomazini, A., Amaral, D. T., Murakami, M. T., \& Viviani, V. R. (2020). Luciferase isozymes from the Brazilian Aspisoma lineatum (Lampyridae) firefly: origin of efficient $\mathrm{pH}$-sensitive lantern luciferases from fat body $\mathrm{pH}$-insensitive ancestors. Photochemical \& Photobiological Sciences, 19(12), 1750-1764.

Chen, Q., Wen, M., Li, J., Zhou, H., Jin, S., Zhou, J. J., ... \& Ren, B. (2019). Involvement of heat shock protein 40 in the wing dimorphism of the house cricket Acheta domesticus. Journal of insect physiology, 114, 35-44.

Chénais, B., Caruso, A., Hiard, S., \& Casse, N. (2012). The impact of transposable elements on eukaryotic genomes: from genome size increase to genetic adaptation to stressful environments. Gene, 509(1), 7-15.

Chikhi, R., \& Medvedev, P. (2014). Informed and automated k-mer size selection for genome assembly. Bioinformatics, 30(1), 31-37.

Choi, J. Y., \& Lee, Y. C. G. (2020). Double-edged sword: The evolutionary consequences of the epigenetic silencing of transposable elements. PLoS genetics, 16(7), e1008872.

Cohen, A. C. (1995). Extra-oral digestion in predaceous terrestrial Arthropoda. Annual review of entomology, 40(1), 85-103.

Colepicolo-Neto, P., Bechara, E. J. H., \& Costa, C. (1986). Oxygen toxicity aspects in luminescent and non-luminescent elaterid larvae. Insect biochemistry, 16(2), 381-385. 
Costa, C., Lawrence, J. F., \& Rosa, S. P. (2010). Elateridae Leach, 1815. Handbook of zoology, 4(Part 38), 75-103.

Costa, C.; Vanin, S.A.; Casari, S.A. \& Viviani, V.R. 1999. Larvae of Neotropical Coleoptera. XXVII. Phrixotrix hirtus: immatures, neotenic female, adult male and bionomic data (Phengodinae, Phengodidae, Coleoptera). Ilheringia. Serie Zoologia, 86: 9-28.

Costa, C., Vanin, S. A., \& Neto, P. C. (1986). Larvae of neotropical Coleoptera. XIV: First record of bioluminescence in the Family Staphylinidae (Xantholini). Revista brasileira de entomologia, 30(1), 101-104.

Cox, H., Norris, C., Wu, G., Guan, J., Hessey, S., \& Stace, A. J. (2011). Evidence of zinc superoxide formation in the gas phase: Comparisons in behaviour between ligated $\mathrm{Zn}$ (I/II) and $\mathrm{Cu}(\mathrm{I} / \mathrm{II})$ with regard to the attachment of O 2 or H 2 O. Dalton Transactions, 40(42), 11200-11210.

Day, J. C., Tisi, L. C., \& Bailey, M. J. (2004). Evolution of beetle bioluminescence: the origin of beetle luciferin. Luminescence: The journal of biological and chemical luminescence, 19(1), 8-20.

Emms, D. M., \& Kelly, S. (2015). OrthoFinder: solving fundamental biases in whole genome comparisons dramatically improves orthogroup inference accuracy. Genome Biology, $16(1), 1-14$.

Fallon, T. R., Li, F. S., Vicent, M. A., \& Weng, J. K. (2016). Sulfoluciferin is biosynthesized by a specialized luciferin sulfotransferase in fireflies. Biochemistry, 55(24), 3341-3344.

Fallon, T. R., Lower, S. E., Chang, C. H., Bessho-Uehara, M., Martin, G. J., Bewick, A. J., ... \& Weng, J. K. (2018). Firefly genomes illuminate parallel origins of bioluminescence in beetles. Elife, 7, e36495.

Feder, J. L., \& Velez, S. (2009). Intergenic exchange, geographic isolation, and the evolution of bioluminescent color for Pyrophorus click beetles. Evolution: International Journal of Organic Evolution, 63(5), 1203-1216.

Fedoroff, N. V. (2012). Transposable elements, epigenetics, and genome evolution. Science, 338(6108), 758-767.

Freitas, L., \& Nery, M. F. (2020). Expansions and contractions in gene families of independently-evolved blood-feeding insects. BMC Evolutionary Biology, 20(1), 1-8. 
Fry, B. G., Roelants, K., Champagne, D. E., Scheib, H., Tyndall, J. D., King, G. F., ... \& De La Vega, R. C. R. (2009). The toxicogenomic multiverse: convergent recruitment of proteins into animal venoms. Annual review of genomics and human genetics, 10, 483511.

Fu, J., Posnien, N., Bolognesi, R., Fischer, T. D., Rayl, P., Oberhofer, G., ... \& Bucher, G. (2012). Asymmetrically expressed axin required for anterior development in Tribolium. Proceedings of the National Academy of Sciences, 109(20), 7782-7786.

Fu, X., Li, J., Tian, Y., Quan, W., Zhang, S., Liu, Q., ... \& Hu, J. (2017). Long-read sequence assembly of the firefly Pyrocoelia pectoralis genome. GigaScience, 6(12), gix112.

Fuzita, F. J., Pinkse, M. W., Patane, J. S., Verhaert, P. D., \& Lopes, A. R. (2016). High throughput techniques to reveal the molecular physiology and evolution of digestion in spiders. BMC genomics, 17(1), 1-19.

Glaser, R. L., Hickey, A. J., Chotkowski, H. L., \& Chu-LaGraff, Q. (2003). Characterization of Drosophila palmitoyl-protein thioesterase 1. Gene, 312, 271-279.

Gurevich, A., Saveliev, V., Vyahhi, N., \& Tesler, G. (2013). QUAST: quality assessment tool for genome assemblies. Bioinformatics, 29(8), 1072-1075.

Han, M. V., Demuth, J. P., McGrath, C. L., Casola, C., \& Hahn, M. W. (2009). Adaptive evolution of young gene duplicates in mammals. Genome research, 19(5), 859-867.

Han, M. V., Thomas, G. W., Lugo-Martinez, J., \& Hahn, M. W. (2013). Estimating gene gain and loss rates in the presence of error in genome assembly and annotation using CAFE 3. Molecular biology and evolution, 30(8), 1987-1997.

Hanrahan, S. J., \& Johnston, J. S. (2011). New genome size estimates of 134 species of arthropods. Chromosome Research, 19(6), 809-823.

Harrison, J. F., Greenlee, K. J., \& Verberk, W. C. (2018). Functional hypoxia in insects: definition, assessment, and consequences for physiology, ecology, and evolution. Annual Review of Entomology, 63, 303-325.

Hazzouri, K. M., Sudalaimuthuasari, N., Kundu, B., Nelson, D., Al-Deeb, M. A., Le Mansour, A., ... \& Amiri, K. M. (2020). The genome of pest Rhynchophorus ferrugineus reveals gene families important at the plant-beetle interface. Communications biology, $3(1), 1-14$. 
Hill, D. P., Berardini, T. Z., Howe, D. G., \& Van Auken, K. M. (2010). Representing ontogeny through ontology: a developmental biologist's guide to the gene ontology. Molecular Reproduction and Development: Incorporating Gamete Research, 77(4), 314329.

Huang, X., \& Madan, A. (1999). CAP3: A DNA sequence assembly program. Genome research, 9(9), 868-877.

Jacobson, M. (2012). Insect sex pheromones. Elsevier.

Jiao, C., Sørensen, I., Sun, X., Sun, H., Behar, H., Alseekh, S., ... \& Rose, J. K. (2020). The Penium margaritaceum genome: Hallmarks of the origins of land plants. Cell, 181(5), 1097-1111.

Jin, J. J., Yu, W. B., Yang, J. B., Song, Y., Depamphilis, C. W., Yi, T. S., \& Li, D. Z. (2020). GetOrganelle: a fast and versatile toolkit for accurate de novo assembly of organelle genomes. Genome biology, 21(1), 1-31.

Kanie, S., Nishikawa, T., Ojika, M. \& Oba, Y. One-pot non-enzymatic formation of firefly luciferin in a neutral buffer from p-benzoquinone and cysteine. Sci. Rep. 6, 24794 (2016).

Kidwell, M. G. (2002). Transposable elements and the evolution of genome size in eukaryotes. Genetica, 115(1), 49-63.

Langmead, B., \& Salzberg, S. L. (2012). Fast gapped-read alignment with Bowtie 2. Nature methods, 9(4), 357.

Lechner, M., Marz, M., Ihling, C., Sinz, A., Stadler, P. F., \& Krauss, V. (2013). The correlation of genome size and DNA methylation rate in metazoans. Theory in Biosciences, 132(1), 47-60.

Li, G., Chen, X., Li, B., Zhang, G., Li, Y., \& Wu, J. (2016). Binding properties of general odorant binding proteins from the oriental fruit moth, Grapholita molesta (Busck)(Lepidoptera: Tortricidae). PLoS One, 11(5), e0155096.

Li, H., Handsaker, B., Wysoker, A., Fennell, T., Ruan, J., Homer, N., ... \& Durbin, R. (2009). The sequence alignment/map format and SAMtools. Bioinformatics, 25(16), 2078-2079.

Liu, G. C., Dong, Z. W., He, J. W., Zhao, R. P., Wang, W., \& Li, X. Y. (2017). Genome size of 14 species of fireflies (Insecta, Coleoptera, Lampyridae). Zoological research, 38(6), 449. 
Lousa, C. D. M., van Roermund, C. W., Postis, V. L., Dietrich, D., Kerr, I. D., Wanders, R. J., .. \& Theodoulou, F. L. (2013). Intrinsic acyl-CoA thioesterase activity of a peroxisomal ATP binding cassette transporter is required for transport and metabolism of fatty acids. Proceedings of the National Academy of Sciences, 110(4), 1279-1284.

Lower, S. S., Johnston, J. S., Stanger-Hall, K. F., Hjelmen, C. E., Hanrahan, S. J., Korunes, K., \& Hall, D. (2017). Genome size in North American fireflies: substantial variation likely driven by neutral processes. Genome biology and evolution, 9(6), 1499-1512.

Marçais, G., \& Kingsford, C. (2011). A fast, lock-free approach for efficient parallel counting of occurrences of k-mers. Bioinformatics, 27(6), 764-770.

McKinley, C. N., \& Lower, S. E. (2020). Comparative transcriptomics reveals gene families associated with predatory behavior in Photuris femme fatale fireflies. Genes, 11(6), 627.

Mendivil, O. R. \& Ferrier, D. E. K. (2012) Mechanisms of Gene Duplication and Translocation and Progresstowards Understanding Their Relative Contributions to Animal Genome Evolution. Int. J. Evol. Biol., 2012, 1-10.

Minh, B. Q., Schmidt, H. A., Chernomor, O., Schrempf, D., Woodhams, M. D., Von Haeseler, A., \& Lanfear, R. (2020). IQ-TREE 2: New models and efficient methods for phylogenetic inference in the genomic era. Molecular biology and evolution, 37(5), $1530-1534$.

Niwa, K., Nakamura, M., \& Ohmiya, Y. (2006). Stereoisomeric bio-inversion key to biosynthesis of firefly D-luciferin. FEBS letters, 580(22), 5283-5287.

Oba, Y., Mori, N., Yoshida, M., \& Inouye, S. (2010). Identification and characterization of a luciferase isotype in the Japanese firefly, Luciola cruciata, involving in the dim glow of firefly eggs. Biochemistry, 49(51), 10788-10795.

O’Connell, J., Schulz-Trieglaff, O., Carlson, E., Hims, M. M., Gormley, N. A., \& Cox, A. J. (2015). NxTrim: optimized trimming of Illumina mate pair reads. Bioinformatics, 31(12), 2035-2037.

O’Keefe, S. T., Arnett, R. H., Thomas, M. C., Skelley, P. E., \& Frank, J. H. (2002). Phengodidae LeConte 1861 (pp. 181-186). Boca Raton: CRC Press LLC.

Oba, Y., Branham, M. A., \& Fukatsu, T. (2011). The terrestrial bioluminescent animals of Japan. Zoological science, 28(11), 771-789. 
Oba, Y., Yoshida, N., Kanie, S., Ojika, M., \& Inouye, S. (2013). Biosynthesis of Firefly Luciferin in Adult Lantern: Decarboxylation of $\square$-Cysteine is a Key Step for Benzothiazole Ring Formation in Firefly Luciferin Synthesis. PLoS One, 8(12), e84023.

Palmer, M., \& Petitpierre, E. (1996). Relationship of genome size to body size in Phylan semicostatus (Coleoptera: Tenebrionidae). Annals of the Entomological Society of America, 89(2), 221-225.

Petitpierre, E., Segarra, C., \& Juan, C. (1993). Genome size and chromosomal evolution in leaf beetles (Coleoptera, Chrysomelidae). Hereditas, 119(1), 1-6.

Quesneville, H. (2020). Twenty years of transposable element analysis in the Arabidopsis thaliana genome. Mobile DNA, 11(1), 1-13.

Rane, R. V., Ghodke, A. B., Hoffmann, A. A., Edwards, O. R., Walsh, T. K., \& Oakeshott, J. G. (2019). Detoxifying enzyme complements and host use phenotypes in 160 insect species. Current opinion in insect science, 31, 131-138.

Rather, M. A., \& Dhandare, B. C. (2019). Genome-Wide identification of doublesex and Mab-3-Related transcription factor (DMRT) genes in nile tilapia (Oreochromis niloticus). Biotechnology Reports, 24, e00398.

Rech, G. E., Bogaerts-Marquez, M., Barrón, M. G., Merenciano, M., Villanueva-Cañas, J. L., Horváth, V., ... \& González, J. (2019). Stress response, behavior, and development are shaped by transposable element-induced mutations in Drosophila. PLoS genetics, 15(2), e1007900.

RepeatMasker 4.0.5. http://www.repeatmasker.org. Accessed 20 May 2019.

RepeatModeler. http://www.repeatmasker.org. Accessed 28 May 2019.

Rosa, S. P. (2010). Second record of bioluminescence in larvae of Xantholinus Dejean (Staphylinidae, Xantholinini) from Brazil. Revista Brasileira de Entomologia, 54(1), 147-148.

Sanderson, M. J. (2003). r8s: inferring absolute rates of molecular evolution and divergence times in the absence of a molecular clock. Bioinformatics, 19(2), 301-302.

Schmieder, R., \& Edwards, R. (2011). Fast identification and removal of sequence contamination from genomic and metagenomic datasets. PloS one, 6(3), e17288. 
Shoji, K., Kiuchi, T., Hara, K., Kawamoto, M., Kawaoka, S., Arimura, S. I., ... \& Katsuma, S. (2013). Characterization of a novel chromodomain-containing gene from the silkworm, Bombyx mori. Gene, 527(2), 649-654.

Simão, F. A., Waterhouse, R. M., Ioannidis, P., Kriventseva, E. V. \& Zdobnov, E. M. BUSCO: assessing genome assembly and annotation completeness with single-copy orthologs. Bioinformatics. 31, 3210-3212 (2015).

Strause, L. G., \& DeLuca, M. (1981). Characteristics of luciferases from a variety of firefly species: evidence for the presence of luciferase isozymes. Insect Biochemistry, 11(4), 417-422.

Tainer, J. A., Getzoff, E. D., Richardson, J. S., \& Richardson, D. C. (1983). Structure and mechanism of copper, zinc superoxide dismutase. Nature, 306(5940), 284-287.

Talla, V., Suh, A., Kalsoom, F., Dincă, V., Vila, R., Friberg, M., ... \& Backström, N. (2017). Rapid increase in genome size as a consequence of transposable element hyperactivity in wood-white (Leptidea) butterflies. Genome Biology and Evolution, 9(10), 2491-2505.

Ting, C. T., Tsaur, S. C., Sun, S., Browne, W. E., Chen, Y. C., Patel, N. H. \& Wu, C.I. (2004). Gene duplication and speciation in Drosophila: Evidence from the Odysseus locus. Proc. Natl. Acad. Sci. USA, 101, 12232-12235.

Trimmer, B. A., Aprille, J. R., Dudzinski, D. M., Lagace, C. J., Lewis, S. M., Michel, T., ... \& Zayas, R. M. (2001). Nitric oxide and the control of firefly flashing. Science, 292(5526), 2486-2488.

Van de Peer, Y., Maere, S. \& Meyer, A. (2009) The evolutionary significance of ancient genome duplications. Nat. Rev. Genet., 10, 725-732.

Viviani, V. R. (2002). The origin, diversity, and structure function relationships of insect luciferases. Cellular and Molecular Life Sciences CMLS, 59(11), 1833-1850.

Viviani, V. R., \& Becham, E. J. (1993). Biophysical and biochemical aspects of phengodid (railroad $\square$ worm) bioluminescence. Photochemistry and photobiology, 58(4), 615-622.

Viviani, V. R., \& Bechara, E. J. (1995). Bioluminescence of Brazilian fireflies (Coleoptera: Lampyridae): spectral distribution and $\mathrm{pH}$ effect on luciferase $\square$ elicited colors. Comparison with elaterid and phengodid luciferases. Photochemistry and photobiology, 62(3), 490-495. 
Viviani, V. R., \& Bechara, E. J. (1997). Bioluminescence and biological aspects of Brazilian railroad-worms (Coleoptera: Phengodidae). Annals of the Entomological Society of America, 90(3), 389-398.

Viviani, V. R., Bechara, E. J., \& Ohmiya, Y. (1999). Cloning, sequence analysis, and expression of active Phrixothrix railroad-worms luciferases: relationship between bioluminescence spectra and primary structures. Biochemistry,38(26), 8271-8279.

Viviani, V. R., Bevilaqua, V. R., de Souza, D. R., Pelentir, G. F., Kakiuchi, M., \& Hirano, T. (2021). A very bright far-red bioluminescence emitting combination based on engineered railroad worm luciferase and 6'-Amino-Analogs for bioimaging purposes. International Journal of Molecular Sciences, 22(1), 303.

Viviani, V. R., Carmargo, I. A., \& Amaral, D. T. (2013). A transcriptional survey of the cDNA library of Macrolampis sp2 firefly lanterns (Coleoptera: Lampyridae). Comparative Biochemistry and Physiology Part D: Genomics and Proteomics, 8(1), 8285.

Viviani, V. R., Joaquim da Silva Neto, A., \& Ohmiya, Y. (2004). The influence of the region between residues 220 and 344 and beyond in Phrixotrix railroad worm luciferases green and red bioluminescence. Protein Engineering Design and Selection, 17(2), 113-117.

Viviani, V. R., Okawachi, F. M., Scorsato, V., \& Abdalla, F. C. (2008). CCD imaging of basal bioluminescence in larval fireflies: clues on the anatomic origin and evolution of bioluminescence. Photochemical \& Photobiological Sciences, 7(4), 448-452.

Viviani, V. R., \& Ohmiya, Y. (2000). Bioluminescence color determinants of Phrixothrix railroad $\square$ worm luciferases: chimeric luciferases, site $\square$ directed mutagenesis of Arg 215 and guanidine effect. Photochemistry and photobiology, 72(2), 267-271.

Viviani, V., Uchida, A., Suenaga, N., Ryufuku, M., \& Ohmiya, Y. (2001). Thr226 is a key residue for bioluminescence spectra determination in beetle luciferases. Biochemical and biophysical research communications, 280(5), 1286-1291.

Vongsangnak, W., Chumnanpuen, P., \& Sriboonlert, A. (2016). Transcriptome analysis reveals candidate genes involved in luciferin metabolism in Luciola aquatilis (Coleoptera: Lampyridae). PeerJ, 4, e2534. 
Vurture, G. W., Sedlazeck, F. J., Nattestad, M., Underwood, C. J., Fang, H., Gurtowski, J., \& Schatz, M. C. (2017). GenomeScope: fast reference-free genome profiling from short reads. Bioinformatics, 33(14), 2202-2204.

Walter, A., Bechsgaard, J., Scavenius, C., Dyrlund, T. S., Sanggaard, K. W., Enghild, J. J., \& Bilde, T. (2017). Characterisation of protein families in spider digestive fluids and their role in extra-oral digestion. BMC genomics, 18(1), 1-13.

Walker, A. A., Weirauch, C., Fry, B. G., \& King, G. F. (2016). Venoms of heteropteran insects: a treasure trove of diverse pharmacological toolkits. Toxins, 8(2), 43.

Wang, P., Luo, Y., Huang, J., Gao, S., Zhu, G., Dang, Z., ... \& Chen, Y. (2020). The genome evolution and domestication of tropical fruit mango. Genome biology, 21(1), 1-17.

Wing, S. R. (1984). A spate of glow-worms (Coleoptera: Phengodidae). Entomological news, 95, 55-57.

Wittmer, W. (1976). Arbeiten zu einer Revision der Familie Phengodidae (Coloptera). Entomol Arb Mus G Frey Tutzing Munch.

Wu, Y., Liu, Y. Y., \& Chen, X. S. (2019). Whole-genome identification of chemosensory genes in two sister blister beetles provides insights into chemosensory evolution.

Xue, H. J., Niu, Y. W., Segraves, K. A., Nie, R. E., Hao, Y. J., Zhang, L. L., ... \& Yang, X. K. (2020). The draft genome of the specialist flea beetle Altica viridicyanea (Coleoptera: Chrysomelidae) provides insight into host plant specialization.

Ye, J., Zhang, Y., Cui, H., Liu, J., Wu, Y., Cheng, Y., ... \& Shi, C. (2018). WEGO 2.0: a web tool for analyzing and plotting GO annotations, 2018 update. Nucleic acids research, 46(W1), W71-W75.

Yuvaraj, J. K., Andersson, M. N., Zhang, D. D., \& Löfstedt, C. (2018). Antennal transcriptome analysis of the chemosensory gene families from Trichoptera and basal Lepidoptera. Frontiers in physiology, 9, 1365.

Zeng, B., Huang, Y., Xu, J., Shiotsuki, T., Bai, H., Palli, S. R., ... \& Tan, A. (2017). The FOXO transcription factor controls insect growth and development by regulating juvenile hormone degradation in the silkworm, Bombyx mori. Journal of Biological Chemistry, 292(28), 11659-11669. 
Zhang, R., He, J., Dong, Z., Liu, G., Yin, Y., Zhang, X., ... \& Li, X. (2020). Genomic and experimental data provide new insights into luciferin biosynthesis and bioluminescence evolution in fireflies. Scientific reports, 10(1), 1-19.

Zhang, W., Zhang, X., Li, K., Wang, C., Cai, L., Zhuang, W., ... \& Liu, X. (2018). Introgression and gene family contraction drive the evolution of lifestyle and host shifts of hypocrealean fungi. Mycology, 9(3), 176-188.

Zhu, F., Moural, T. W., Nelson, D. R., \& Palli, S. R. (2016). A specialist herbivore pest adaptation to xenobiotics through up-regulation of multiple Cytochrome P450s. Scientific reports, 6(1), 1-10.

\section{Figures captions}

Figure 1. Phylogenetic context of Elateriformia. a. Phrixothrix hirtus railroad worm. b Phylogenetic tree depicting the relationship within Elateroidea bioluminescent species, with Agrilus planipennis (Buprestidae) as outgroup.

Figure 2. Barplot of shared and unique orthologues among Elateriformia genomes. In blue, the number of single-copy genes shared among the species in comparison to multiple copies orthogroups (in black).

Figure 3. Summary tree shows the inference of the gene family evolution based on the expansion (+ blue) and contraction (- red) gene families in Elateriformia. The number between parentheses represents the number of rapidly evolving gene families. 


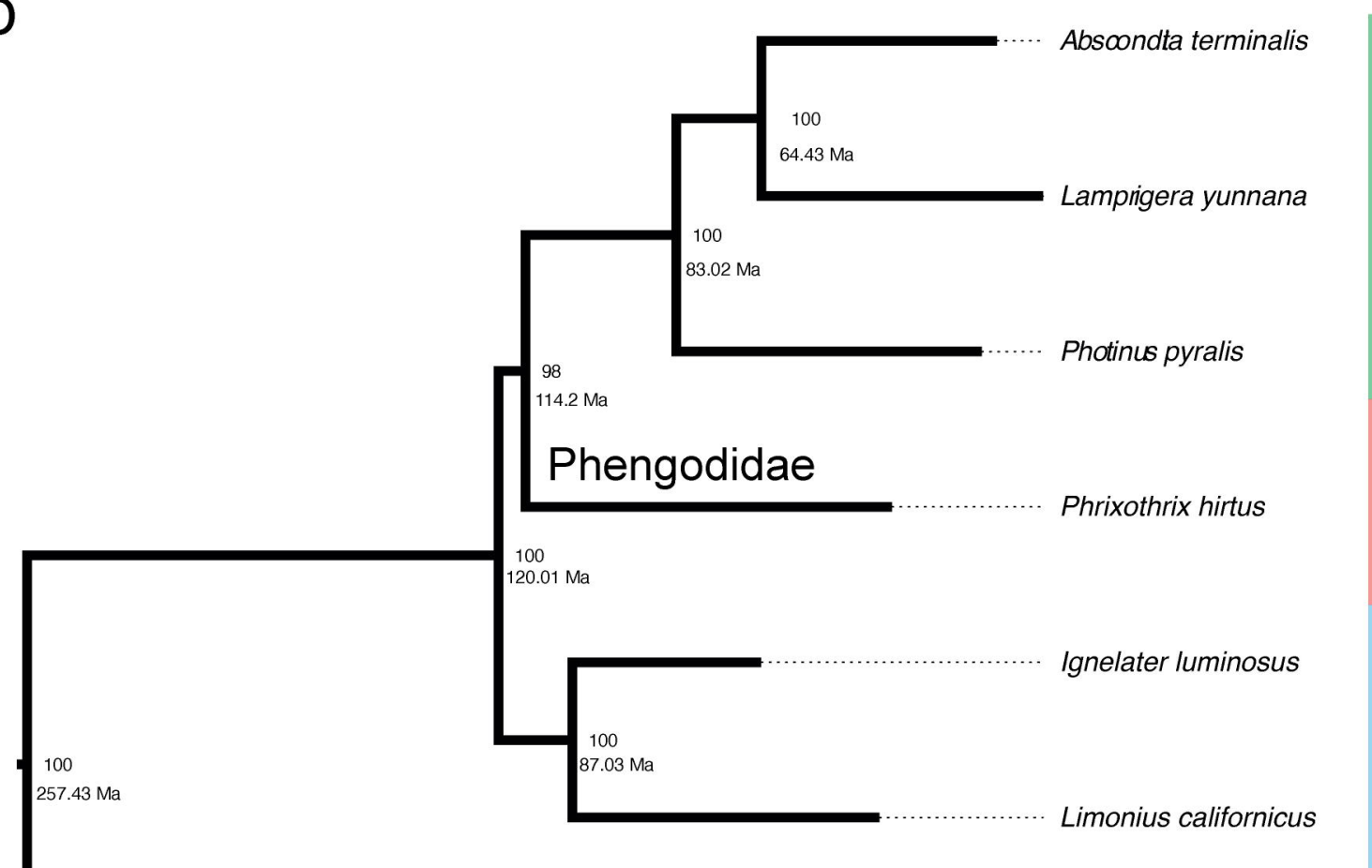

$\frac{m}{0}$

$\stackrel{\overline{0}}{\overrightarrow{0}}$

음.

응 윽

T

$\stackrel{\square}{\square}$

응.

ब. 

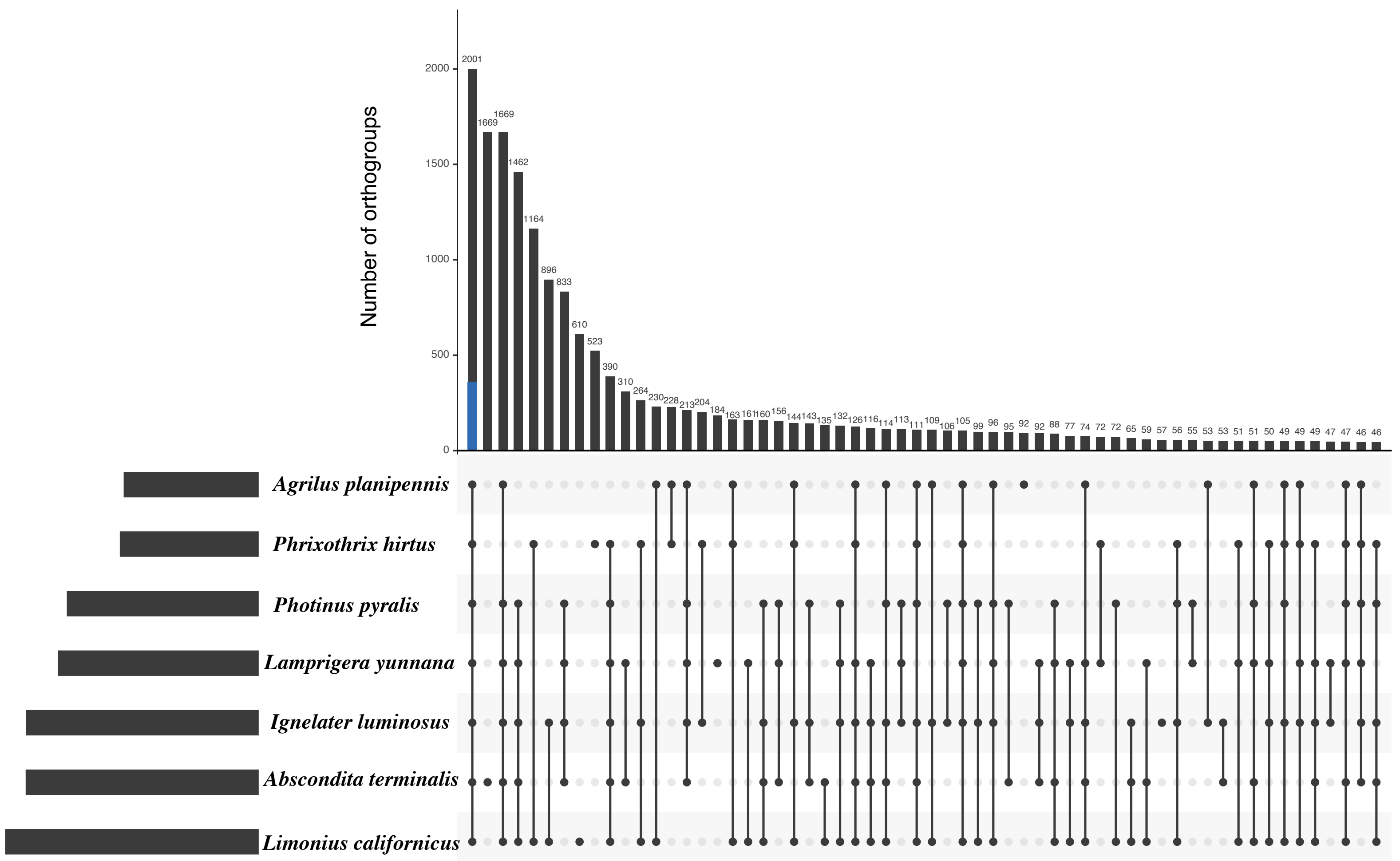


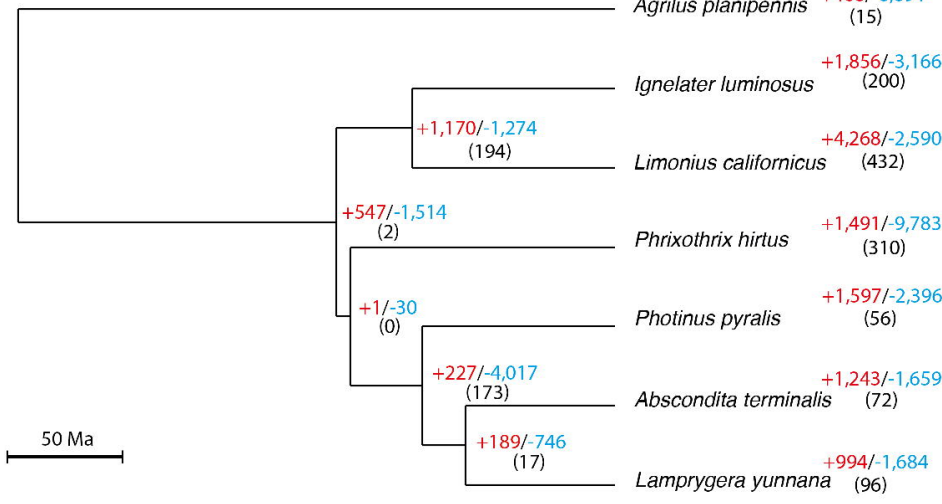

